\title{
Vaccinia Virus H7-Protein Organizes the Viral Scaffold Protein-Essential for Membrane and Virus Assembly
}

\section{Susanne Tonnemacher}

Paul Ehrlich Institut

\section{Marcia Folly-Klan}

Institut Pasteur

Anastasia Gazi

Institut Pasteur

\section{Simon Schäfer}

Paul Ehrlich Institut

\section{Esthel Penard \\ Institut Pasteur}

\section{Regina Eberle}

Paul Ehrlich Institut

Renate Kunz

University of Ulm

Paul Walther

University of Ulm

Jacomine Krijnse Locker ( $\square$ Jacomina.KrijnseLocker@pei.de )

Paul Ehrlich Institut

\section{Research Article}

Keywords: giantviruses, poxvirus, electron microscopy, membrane assembly

Posted Date: March 3rd, 2022

DOI: https://doi.org/10.21203/rs.3.rs-1342101/v1

License: (c) (i) This work is licensed under a Creative Commons Attribution 4.0 International License.

Read Full License 


\section{Abstract}

Viruses of the giant virus family are characterized by a structurally conserved scaffold-capsid protein that shapes the icosahedral virion. The vaccinia virus (VACV) scaffold protein D13, however, transiently shapes the newly assembled viral membrane in to a sphere and is absent from the mature brick-shaped virion. In infected cells D13, a 62kDa polypeptide, forms trimers that arrange in hexamers and a honeycomb like lattice. Membrane association of the D13-lattice may be mediated by A17, an abundant 21kDa viral membrane protein. Whether membrane binding mediates the formation of the honey-comb lattice or if other factors are involved, remains elusive. Here we show that $\mathrm{H7}$, a $17 \mathrm{kD}$ a protein conserved among poxviruses, mediates proper formation of D13-hexamers, and hence the honey comb lattice and spherical immature virus. Without $\mathrm{H} 7$ synthesis $\mathrm{D} 13$ trimers organize into a regular network, composed of short D13 tubes of discrete length that are tightly associated with the endoplasmic reticulum (ER). The data show an unexpected role for $\mathrm{H} 7$ in D13 organization and imply that formation of the honey-comb, hexagonal, lattice is essential for VACV membrane assembly and production of infectious progeny. The data are discussed with respect to scaffold proteins of other giant viruses.

\section{Significance Statement}

The first giant virus to be identified was Mimivirus and new members of this family are continuously discovered. Beside their striking size, most of these viruses acquire an icosahedral shape, mediated by an abundant viral protein on the surface of the virion. The structure of this protein is highly conserved and mediates the formation of a characteristic honey-comb like lattice that may be stabilized by minor viral proteins. We now show that vaccinia virus requires the synthesis of a small viral protein, $\mathrm{H7}$, to form the typical scaffold-lattice on the surface of the viral membrane. Our data imply yet another conserved feature of giant viruses and may contribute to understanding where these large viruses came from.

\section{Introduction}

Enveloped viruses acquire their membrane from the host, via budding at cellular membranes or by wrapping, acquiring a single or double membrane(s), respectively (Welsch et al., 2007). However, for the large DNA-virus vaccinia virus (VACV), member of the poxviridae, the origin and biogenesis of its membrane remains controversial until this date.

By electron microscopy (EM) newly synthesized VACV membranes first appear as short, half-moon shaped units, the crescents, that grow into membrane spheres, the immature virus (IV). Upon DNA-uptake the IV matures in to the brick-shaped mature virus (MV) that is infectious (Moss, 2015). The formation of the crescent membrane and spherical IVs critically depends on the viral scaffold protein, the gene product of $\mathrm{D} 13$, a $62 \mathrm{kDa}$ protein. It assembles on the convex side of the growing viral membrane mediating curvature and in the absence of D13 crescents, IVs and consequently MVs, fail to form (Zhang and Moss, 1992). In infected cells D13 forms a regular structure on the surface of the viral membrane. It is composed of hexagons of D13 trimers, arranged in a high-order lattice resembling a honey comb (Heuser, 
2005, Szajner et al., 2005). Based on electron tomography (ET) we proposed that the honey-comb lattice preassembles in the cytoplasm and then recruits vesicles of roughly $50 \mathrm{~nm}$ in diameter containing viral membrane proteins (Chlanda et al., 2009). The vesicles are opened by membrane rupture and contribute to the formation of IV-membrane, an open lipid bilayer shaped by D13 on its convex side. Association of D13 with viral membranes is mediated by the gene product of $A 17$, a major viral membrane protein of $21 \mathrm{kDa}$ (Bisht et al., 2009; Garriga et al., 2018); it is synthesized in the endoplasmic reticulum and locates to the crescent-, IV- and MV-membrane. When expressed in vitro D13 readily forms trimers; under low salt conditions a higher-order structure resembling a honey-comb-like lattice can be observed but its structure is too heterogeneous to be analyzed in detail (Hyun et al., 2010). Indeed, one study suggested that in infected cells lattice-formation requires the synthesis of viral late proteins (Szajner et al., 2005).

Conditional lethal mutants of VACV provide significant insight into the role of viral proteins in its replicative cycle, assembly and membrane biogenesis (Moss, 2015). Five VACV proteins, the gene products of $A 6, A 11, H 7, L 2$ and A30,5, collectively called virus membrane associated proteins (VMAPs), seem required for viral membrane biogenesis and the formation of infectious virus. We recently proposed, that the VMAP A11 is required for formation of the $50 \mathrm{~nm}$ vesicles, that are recruited to the D13 honey comb lattice to mediate viral membrane assembly. In the absence of A11, D13 accumulates in confined areas that are surrounded by closed ER-cisternae (Suarez et al., 2017); vesicle-formation and membrane opening seemed impaired, as assessed by EM-immuno-labeling and ET. Crescent-, IV- and MV-formation was absent, supporting our model that vesicle formation and membrane opening are necessary for VACV membrane-biogenesis (Chlanda et al., 2009).

When the expression of $\mathrm{H} 7$, another VMAP, is blocked, IV- and MV-formation is absent as well (Satheshkumar et al., 2009) arguing for an important role of this protein in virus assembly. The H7 sequence predicts a $17 \mathrm{kD}$ a protein that by $\mathrm{X}$-ray crystallography was shown to consist of seven a-helical domains, three $\beta$-strands and a 29 amino acids long $C$-terminal flexible tail. The seventh $a$-helical domain predicted a putative PX domain, involved in binding to phosphoinositides. Indeed, in dot blot experiments H7 was shown to bind to both PI3P and PI4P (Kolli et al., 2015).

The present study was conducted to further investigate the role of $\mathrm{H} 7 \mathrm{in}$ VACV-assembly. We confirm that $\mathrm{H} 7$ is required for IV- and MV-formation in HeLa cells. Surprisingly, we find that in the absence of H7 D13 collects in structures, that are significantly different from its honey-comb like arrangement, and that are intimately associated with the ER. Our data argue for a role of $\mathrm{H} 7$ in D13-lattice formation, thus shedding light on the molecular requirement for their formation.

\section{Results}

\section{Thin section EM confirms that assembly is blocked in the absence of $\mathrm{H7}$}

When $\mathrm{H} 7$ is not synthesized the formation of IVs and MVs is blocked; dense inclusions accumulate collecting viral core proteins that are coated with structures resembling short viral crescents. A second structure accumulates close by, that collects D13 (Satheshkumar et al., 2009). 
Conventional EM of HeLa cells fixed at $12 \mathrm{hrs} \mathrm{post-infection} \mathrm{largely} \mathrm{confirmed} \mathrm{these} \mathrm{observations.} \mathrm{In} \mathrm{the}$ presence of isopropyl-b-D-thiogalactopyranoside (IPTG), structures typical of a wild-type infection (crescents, IVs and MVs) were observed (figure 1A and B). Without H7-synthesis IVs and MVs were absent; large areas of lower electron-density, the VACV-replication site, collected electron-dense aggregates (figure 1C; $\mathrm{Vi}$ ) that were occasionally coated with arcs resembling short crescents (figure 1E). At the periphery of the replication sites a second prominent structure, not described before and absent in the presence of IPTG, was seen. Electron-dense spots were arranged in a regular pattern and associated with membranes reminiscent of the ER (figure 1D; NS).

EM immuno-labeling confirmed the expected localization of VACV proteins in the presence of IPTG; antiD13 to the surface of the IV membrane (figure 2A) and anti-A17 to the membrane of both the IVs (figure $2 \mathrm{~A}$ ) and the MVs (figure 2B). The electron-dense aggregates in the absence of IPTG collected the core protein A3 (not shown) while the short arcs were labeled with anti-D13 and A17 (figure 2D), confirming that they are short crescent-membranes. The network labeled prominently for anti-D13 showing that the scaffold protein accumulated in these aberrant structures. Anti-PDI confirmed that the membranes were derived from the ER (not shown). A17, the binding partner of D13, localized to the surrounding ER membranes (figure $2 \mathrm{C}$ ) where it was not particularly enriched when compared to random pieces of ER (not shown).

The collective data suggested that $\mathrm{H} 7$ was involved in the proper formation of D13 in infected cells, resulting in the dense network associated with the ER. The D13 structures were next analyzed in 3D by ET.

\section{Scanning- and transmission electron tomography reveal an aberrant organization of D13}

Sections, $750 \mathrm{~nm}$ in thickness, of conventionally embedded samples were analyzed by scanning transmission electron tomography (STEM-ET), focusing on the 3D organization of the D13-network structure. In 3D the intimate association of the D13-network with the ER was readily observed (figure 3; movie 1 and 2). The rendering shown in movie 2 illustrated how the ER cisternae surrounded, and moved perpendicular into, the network (figure 3; movie 2). Slice by slice inspection of the tomograms revealed that the spots were connected to each other by thin tubes of distinct length, altogether contributing to the regular network appearance (figure 3 white arrowheads; movie 3). The electron-dense spots were seen to pull on the ER membrane to form a tube, suggesting that the connections were derived from the ERmembrane (figure 3; see also below). Rendering of the electron-dense D13-spots in z, within the 750nm volume of the section, displayed them as short hollow tubes, roughly $22 \mathrm{~nm}$ in width (movie 2 and 3 ). Dual-axis transmission electron tomography of thawed cryo-sections, $150 \mathrm{~nm}$ in thickness, labeled with anti-D13 confirmed first that the scaffold protein accumulated within the network (figure 4 Z:5). Second, the D13-labeled structures showed a massive accumulation of short membrane fragments (figure 4 $Z: 127)$, that appeared as white lines due to the negative contrasting used in this method (figure 4, movie 4). Their abundance strongly suggested these to correspond to the membrane tubes connecting the D13 units shown by STEM-ET. The electron-dense D13-spots were not readily visible in the tomograms of the thawed cryo-sections, likely because of the negative contrasting and the smaller volume analysed. 
The collective 3D-EM data argued that in the absence of H7, D13 formed short hollow tubes of discrete length and diameter, interconnected by short membrane tubes, forming a regular pattern, intimately associated with ER-cisternae. The structure of the D13-spots was next analyzed with a hybrid cryoET method.

\section{Refrozen Tokuyasu sections show D13 trimers but an absence of hexagons.}

Under wild-type infection conditions D13 forms hexamers of D13-trimers. The trimers measure 7 to $9 \mathrm{~nm}$ in diameter, while the hexamers measure roughly 20-22 nm from vertex to vertex, depending on the EM method used (Heuser, 2015; Chlanda et al., 2009). The average width of $22 \mathrm{~nm}$ of the D13-spots, measured in the STEM-tomograms, suggested that D13 might form hexamers but that lattice formation was impaired in the absence of H7. However, the contrasting used displayed D13 as (uniform) electrondense dots and failed to reveal trimers or hexamer-formation.

The technique of refrozen Tokuyasu sections (Bos et al., 2014) was applied to analyse the D13-structure. Cryo-sections, 70nm in thickness, were thawed and labeled with anti-D13, the sections were vitrified by plunge-freezing and imaged by cryoET. In the presence of IPTG the honey-comb lattices were readily observed in 3D, located on the surface of the IVs or as small discrete patches next to the IVs, as shown before (figure 5A, movie 5; Chlanda et al., 2009). By inverting the contrast, the organization of D13 trimers into hexamers, together forming the honey comb lattice was readily seen (figure 5B and C, movie 5). In the absence of IPTG the D13-network could not be unequivocally identified without prior immunolabeling. Areas labeled for D13 on the surface of the section were subjected to tilt-series-acquisition and structures analyzed (movie 6). Structures with an average diameter of $8-9 \mathrm{~nm}$, were readily observed in these areas (figure 5D and E). By superimposing the known x-ray structure (Garriga et al., 2018) these could be identified as D13-trimers (figure $5 \mathrm{~F}$ ). Although the trimers arranged in a regular pattern, we failed to observe a hexagonal arrangement (figure 5F). Instead D13 collected in a pattern of 3-5 trimers separated from each other by roughly $10 \mathrm{~nm}$ (figure $5 \mathrm{E}$ and F). By cryoET the D13-positive area also displayed many short membrane-tubes but their structure and relation to the D13 units was not readily displayed.

We propose that the electron-dense spots seen by RT-EM are composed of several D13-trimers, lacking the hexagonal arrangement. The trimers are arranged to form a regular pattern in x-y by cryo-ET that form the $\sim 22 \mathrm{~nm}$ electron-dense spots seen by conventional EM and arrange in hollow tubes in z (STEM-ET). What connects that D13-trimers to form a regular pattern remains elusive (see discussion).

\section{Localization of $\mathrm{H7}$}

A putative role for $\mathrm{H} 7$ in proper formation of the $\mathrm{D} 13$ into honey-comb patches was surprising and prompted us to re-investigate its localization in infected cells, previously proposed to be predominantly cytoplasmic (Satheshkumar et al., 2009). We took advantage of the fact that recombinant H7-protein expressed in the presence of IPTG was tagged with an HA-epitope. In addition, infection with VACV-H7ind without IPTG and transfected with HA-tagged full-length $\mathrm{H} 7$ was used to over-express $\mathrm{H} 7$ using a VACV 
synthetic early/late promotor. Transfection efficiency was roughly $90 \%$, based on anti-HA labeling by light microscopy. Expression of the full-length $\mathrm{H} 7$ lead to an efficient rescue of the phenotype observed without $\mathrm{H} 7$-synthesis, showing that transfected $\mathrm{HA}-\mathrm{H} 7$ was functional (supplemental figure 2A). HAtagged $\mathrm{H7}$ expressed in the presence of IPTG or upon transfection displayed a general cytoplasmic labeling both by LM (supplemental figure 1) and EM (data not shown) with no concentration on, or close to, the viral membranes. While surprising, they confirm previous results (Satheshkumar et al., 2009).

\section{Residues required for assembly}

Expression of HA-tagged $\mathrm{H} 7$ in trans produced a phenotype indistinguishable from infection in the presence of IPTG; infected/transfected cells displayed the full complement of crescents, IVs and MVs, while the electron-dense virosomes and ER-associated networks were absent (supplemental figure 2A; Table I). The efficient rescue of the H7-phenotype prompted us to analyse residues required for membrane assembly. While single residues within $\mathrm{H} 7$ required for infectivity were previously analysed (Kolli et al., 2015), we focused on rescue of assembly scoring for IV and MV-formation by EM. In first instance we used truncated constructs of $\mathrm{H7}$, expressing amino acid 1 to 114 (N-terminus) or 119 to 146 (C-terminus) of $\mathrm{H7}$. Both constructs failed to rescue assembly, implying that both the $\mathrm{N}$ - and $\mathrm{C}$-terminal part of the protein is essential for D13 organization (Table I).

Previous experiments implied an important role for positively charged amino acids of the $\mathrm{H} 7$ protein for infectivity (Kolli et al., 2015). Specifically, substituting the lysins at positions 108, 128 and 143 individually, affected the production of infectious virus, as well as a triple mutant substituting lysine 108, arginine 109 and lysine 112. The latter three amino acids map in the putative PX, PIP-binding domain of $\mathrm{H} 7$. We substituted all positively charged amino acids individually, expressed the mutant proteins as described above and quantified the different viral forms, IVs and MVs (Table I). None of the single point mutations affected assembly and the full complement of viral forms was made to the same extend in transfected cells (Table I; supplemental figure 2B and data not shown). Mutating all three positively charged amino acids in helical domain 7 failed to rescue assembly (Table l; supplemental figure 2D). In addition, a double mutant substituting lysine 128 and 143 also affected assembly, IVs were observed, whereas MVs were absent (Table l; supplemental figure 2C).

The data thus show, and confirm, an important role for the helical domain 7, in particular its three positively charges amino acids, its putative phosphoinositide binding domain.

\section{Discussion}

Although biogenesis of the VACV-membrane remains controversial until this date, viral proteins involved have been identified thanks to conditional deletion mutants (Moss, 2015). A recent focus is a set of less abundant viral proteins, the VMAPs, that are important for viral membrane assembly and thus the production of infectious progeny. Thus, we recently analyzed the role of A11 by 3D EM; in its absence D13 collects in cytoplasmic patches, seemingly with a honey comb arrangement, surrounded by ERcisternae (Suarez et al., 2017). We concluded that A11 might be involved in the formation of the $50 \mathrm{~nm}$ 
vesicles, which we predict in our model to be the precursors of VACV membrane biogenesis, and in their membrane rupture. The present study asked whether the gene product of H7, a 17kDa VMAP, could also mediate membrane rupture. In its absence short crescents with open ends are formed that are associated with viral core proteins suggesting a role in membrane elongation rather than rupture. This discriminates $\mathrm{H} 7$ from $\mathrm{A} 11$ where crescent formation and membrane rupture is absent altogether. Our data suggest that without H7, D13 is able to form trimers, but the formation of hexamers and the high order honey combstructure, is impaired. This places $\mathrm{H} 7$ into the first viral factor required for D13 organization in infected cells and shows that this organization is essential for proper viral membrane assembly.

The aberrant structures where D13 accumulate in the absence of $\mathrm{H} 7$ are intriguing. It shows a high affinity of D13 for ER membranes. These ER membranes are not particularly enriched for A17, the membrane protein proposed to mediate membrane binding of D13. This observation is similar to the phenotype seen in the absence of A11 expression, where D13 accumulations are surrounded by ERcisternae that are not enriched in A17 (Suarez et al., 2017). A striking feature of the D13 network formed in the absence of $\mathrm{H7}$, is its regular pattern. Although hexamer formation was impaired, D13-trimers were arranged in regular pattern composed of several trimers. It suggests that the individual trimers are interconnected, but the nature of such putative connections remains elusive. D13 protein assemblies composed of several trimers, formed hollow tubes that, in turn, were interconnected by thin membrane tubes with a regular shape and length. It suggests that D13 has an intrinsic ability to recruit, bind to and modify membranes of the ER, although at present is it not clear if D13 is the sole factor mediating this.

With an important role in both membrane elongation and D13 organization, the cytoplasmic localization of $\mathrm{H7}$, without co-localization with viral structures, is surprising. The lipidome of VACV shows an enrichment for phosphoinositides (PIs; Krijnse Locker et al., 2013). A possible scenario is that $\mathrm{H7}$ transiently binds via its PX-domain to a specific PI located to the viral growing membrane, mediating its elongation. $\mathrm{H} 7$ also seems to play a role in $\mathrm{D} 13$ lattice-formation, promoting hexamer formation and likely trimer/trimer interactions. Failure to localize $\mathrm{H} 7$ to the D13-lattice could indicate that its HA-tag is inaccessible to the antibody used or that it is a minor constituent of the viral honey-comb lattice. Indeed, the capsid proteins of other viruses of the giant virus-family, that show structural homology to D13 and also arrange in hexagonal arrays on the surface of mature virions, may be stabilized by minor viral proteins (reviewed in Xiao and Rossmann, 2011). A final scenario is that proper organization (in a honeycomb like lattice) of membrane-associated D13 mediated by $\mathrm{H} 7$ facilitates membrane elongation. Short crescents are produced with a proper D13 lattice that fail to grow since the majority of D13 is misorganized and not available for membrane biogenesis. Experiments are currently carried out to test these hypotheses. However, due to its non-co-localization with typical viral structures involved in VACVassembly we did not attempt to analyse $\mathrm{H} 7$ interacting partners.

Altogether, $\mathrm{H} 7$ plays an important role in the cytoplasmic organization of $\mathrm{D} 13$ that we show to be essential for proper membrane assembly of VACV. As small crescents are formed, $\mathrm{H} 7$ is not required for membrane rupture perse. For the latter A11 is a more likely candidate as no open membranes are observed in its absence. Finally, our data also suggest an intrinsic affinity of D13 for membranes of the 
ER, not necessarily involving its binding partner A17. This affinity may lead to a reorganization of the ERmembrane that, in the absence of $\mathrm{H7}$, results in the formation of thin membrane tubes and, we speculate, in the recruitment of $50 \mathrm{~nm}$ vesicles under wild-type infection conditions. Further studies are now on the way to elucidate the role of other VMAPs, their role in D13 organization and membrane biogenesis.

\section{Materials And Methods}

\section{Cell and virus culture}

HeLa cells (ATTC-CCL-2) and BSC-40 cells (a kind gift of Jason Mercer, university college London, UK) were grown in Dulbecco's modified Eagle's medium (DMEM) containing penicillin and streptomycin and 10\% fetal calf serum. To prepare VACV-H7-ind, a kind gift of Bernie Moss (Satheshkumar et al., 2009), BSC-40 cells were grown to $70 \%$ confluency. Cells were infected in the presence of $100 \mathrm{mM}$ isopropyl-b-Dthiogalactopyranoside (IPTG; Sigma) at low MOI (0.1) and virus harvested 3 days post-infection as described (Jensen et al., 1996). Virus titers were determined by light microscopy by infecting HeLa cells with 2-fold dilutions of concentrated virus in the presence of IPTG and fixed $8 \mathrm{hrs}$ post-infection. The percentage of infected cells was estimated by indirect immunofluorescence using an antibody to A14.

\section{Antibodies}

Antibodies used are described in Suarez et al., 2017. In brief: anti-A14 (Salmons et al., 1997), anti-core (Pedersen et al., 2000), anti-D13 (Sodeik et al., 1994), anti-A17 (raised to amino acid 17-37 of A17; Wallengren et al., 2001) and anti-HA (Sigma). Protein A couple to 10 or $15 \mathrm{~nm}$ gold was purchased from central microscopy core facility (CMC) Utrecht, the Netherlands.

\section{Sample preparation for EM and ET}

Infected cells were processed for EM-immuno-labeling as described in Suarez et al., 2017. In brief, at the indicated time post-infection cells were fixed for $1 \mathrm{hr}$ at room temperature (RT) in $4 \%$ paraformaldehyde (PFA; electron microscopy sciences cat\# 15710) and $0.1 \%$ glutaraldehyde (GA; electron microscopy sciences cat\#16216) in PHEM buffer (60mM Pipes, 25mM HEPES, $10 \mathrm{mM}$ EGTA, $2 \mathrm{mM} \mathrm{MgCl}_{2}, \mathrm{pH}$ 6.9) and kept at $4^{\circ} \mathrm{C}$ in $4 \%$ PFA in PHEM buffer until further processing. For Tokuyasu cryo-sectioning, thawed cryosections were single- or double-labeled as described (Ghosh et al., 2003). Labeled sections were contrasted and dried in $0.4 \%$ uranyl acetate in $2 \%$ methylcellulose. For Epoxy resin embedding all reagent were from Sigma; the fixed cells were, post-fixed with 2,5\% GA, pelleted and mixed 1:1 with 3\% agarose. The agarose was cut and the cubes post-fixed with $1 \% \mathrm{OsO}_{4}$, followed by contrasting with $0.1 \%(\mathrm{w} / \mathrm{v})$ tannic acid in $0.5 \%$ HEPES, pH7.4. After washing twice with $1 \% \mathrm{Na}_{2} \mathrm{SO}_{4}$ and three times with water, the cubes were contrasted with $2 \%$ UA in water, followed by dehydration with increasing concentration of ethanol. Dehydrated sample was infiltrated with increasing concentration of epoxy resin diluted in ethanol, before infiltration with pure resin and polymerization at $65^{\circ} \mathrm{C}$.

Transmission electron microscopy, (cryo-) electron tomography 
Thin $(70 \mathrm{~nm})$ immuno-labeled sections were observed in a Jeol 1400 Flash operated at $80 \mathrm{kV}$ and equipped with a Xarosa camera. Dual-axis tilt series of thawed cryo-sections $150 \mathrm{~nm}$ in thickness labeled for anti-D13 were acquired with a Tecnai (Thermofisher) operated at 200kV with field emission gun using a Fishione dual-axis holder, and equipped with a Gatan Ultrascan 4000 camera. Briefly, grids were mapped using SerialEM and positions of interest stored for semi-automated image acquisition of tilt series increment 1, binning 2 at 19,000 magnification (pixel size $0.37 \mathrm{~nm}$ ) at tilt angles $+/-70$ degrees. For cryoET of vitrified sections, 70nm cryo-sections were placed on holy carbon coated grids (Quantifoil R 2/2; EM services), thawed and immuno-labeled. Sections were washed with PHEM buffer and after brief blotting (3 sec) plunge frozen using EMGP (Leica) and 2sec for post-blotting time. Cryo-tomograms were acquired with a Tecnai F20 TEM operating at 200kV with SerialEM using low-dose mode (Mastronarde, 2005). The Gatan 626 cryo-holder was used at tilt angles -46 to +52 degrees, increment 2 at a magnification of $29 \mathrm{~K}$ (no binning -pixel size $0.3168 \mathrm{~nm}$ using the US4000 CCD Gatan camera). WBP was used with the SIRT like filter of 7 iterations. For STEM tomography, $800 \mathrm{~nm}$ sections of resin-embedded cells were cut and placed on bar grids without formvar coating. Gold particles were placed on both sides of the sections and overlayed with a thin layer of carbon. STEM tilt series were acquired with a Jeol JEM$2100 \mathrm{~F}$ at $200 \mathrm{kV}$ and the tilt angles $-72^{\circ}$ to $+72^{\circ}$ with $1.5^{\circ}$ increment. The tilt series were recorded with the EM-Tools software (TVIPS, Tietz) and each image had 1024x1024 with the pixel size 2.74nm. All tilt series were reconstructed in IMOD using protein A coupled to $10 \mathrm{~nm}$ gold as fiducials. (Kremer et al., 1996). Movies and rendering were made in IMOD and Fiji.

\section{Quantification}

Cryo-tomograms were binned 4 times using the binvol function of IMOD and the Mitchell antialiasing filter before further analysis. Smaller volumes were extracted, and their contrast was inverted using the mrcbyte function of IMOD with the -R flag on, prior to proceed with ChimeraX (Goddard et al., 2007; Pettersen et al., 2021) iso-surface representations (Figure 5F) or ImageJ/Fiji (Schindelin et al., 2012). In the case of infection with IPTG, the scale space filter (Martinez-Sanchez et al., 2014) was used on top to have the honeycomb lattice easily observable in close zoom. Mean lattice distances were calculated by tracing an IMOD model (Figure 5C) and extract their lengths through the imodinfo function. Fiji was used to assay the Delaunay Voronoi mean distance after manual picking of positions of the D13 trimer in the honeycomb lattice of the wild type VACV scaffold and the D13 clusters observed at the H7 mutant (Figure 5B \& E, Supplemental Figure 3) (Schindelin et al., 2012).

\section{Cloning, transfections and light microscopy}

For transfection experiments, constructs of HA-tagged wild-type or mutants $\mathrm{H} 7$ were used. $\mathrm{H} 7$ gene was amplified by PCR using vaccinia virus genomic DNA as template. PCR product was then digested with Notl and Sacl and subcloned into the modified pBluescript II vector (Frischknecht et al., 1999). The same protocol was used for mutant's constructs using strings DNA fragments (Thermofisher) as template for PCR. All constructs were validated by sequencing. HeLa cells grown on glass slides were infected with VACV H7ind in the presence of $100 \mathrm{mM} \mathrm{IPTG}$ and fixed at $12 \mathrm{hrs}$ post-infection with $3 \%$ PFA. Cells were 
permeabilized with TX-100 and triple-labeled with anti-A17, anti-HA and DAPI followed by confocal microscopy. For transfection, $1 \times 10^{5}$ cells were seeded per well of a 24 -well plate. Cells were infected with VACV H7ind without IPTG for one hours at $37^{\circ} \mathrm{C}$. Meanwhile the transfection mix was prepared in Optimem (Gibco), according to the instructions of the manufacturer using $1 \mathrm{mg}$ of plasmid DNA and $1 \mathrm{ml}$ of Lipofectamine 2000 (Invitrogen) per well and the mix incubated for 15 to 30 minutes at RT. After infection cells were washed with serum-free medium and the transfection mix added dropwise to the cells. After incubation for another $4 \mathrm{hrs}$ at $37^{\circ} \mathrm{C}$, the transfection mix was removed and replaced by serum-free medium. Cells were fixed at the indicated times post-infection and processes for conventional EM or for light microscopy.

\section{Declarations}

\section{Acknowledgments and funding sources}

We thank the EM facilities of Institut Pasteur (Paris) and Paul Ehrlich Institute (Langen, Germany) for providing the resources to carry out the experiments, Bernie Moss $(\mathrm{NIH})$ for providing us with the $\mathrm{H} 7$ inducible virus. Part of this work was funded by a joint ANR/DFG grant (Agence national de recherche and Deutsch Forschungs Gemeinschaft- ANR-16-CE92-0008) to MFK and JKL. JKL and ST are funded by the excellence cluster 'Loewe-DRUID' (Landes Offensive zur Entwicklung wissenschaftlich-oekonomischer Excellence- Drug targets against poverty-related and neglected diseases) of the university of Giessen, and the state Hesse, Germany.

\section{Data availability/Availability of Data and Materials statement}

All data generated or analysed during this study are included in this published article and its supplementary information files.

\section{References}

1. Bisht, H., Weisberg, A.S., Szajner, P., and Moss, B. (2009). Assembly and Disassembly of the CapsidLike External Scaffold of Immature Virions during Vaccinia Virus Morphogenesis. J. Virol. 83, 91409150 .

2. Chlanda, P., Carbajal, M.A., Cyrklaff, M., Griffiths, G., and Krijnse-Locker, J. (2009). Membrane Rupture Generates Single Open Membrane Sheets during Vaccinia Virus Assembly. Cell Host Microbe 6, 8190.

3. Frischknecht, F., Moreau, V., Röttger, S., Gonfloni, S., Reckmann, I., Superti-Furga, G., and Way, M. (1999). Actin-based motility of vaccinia virus mimics receptor tyrosine kinase signalling. Nature 401, 926-929.

4. Garriga, D., Headey, S., Accurso, C., Gunzburg, M., Scanlon, M., and Coulibaly, F. (2018). Structural basis for the inhibition of poxvirus assembly by the antibiotic rifampicin. Proc. Natl. Acad. Sci. 115, 8424-8429. 
5. Ghosh, P., Griffith, J., Geuze, H.J., and Kornfeld, S. (2003). Mammalian GGAs act together to sort mannose 6-phosphate receptors. J. Cell Biol. 163, 755-766. Heuser, J. (2005). Deep-etch EM reveals that the early poxvirus envelope is a single membrane bilayer stabilized by a geodetic "honeycomb" surface coat. J. Cell Biol. 169, 269-283.

6. Goddard, T.D., Huang C.C. and Ferrin, T.F. (2007). Visualizing density maps with UCSF Chimera. J. Struct. Biol. 157: 281-287.

7. Hyun, J.-K., Accurso, C., Hijnen, M., Schult, P., Pettikiriarachchi, A., Mitra, A.K., and Coulibaly, F. (2011). Membrane Remodeling by the Double-Barrel Scaffolding Protein of Poxvirus. PLoS Pathog. 7, e1002239.

8. Jensen, O.N., Houthaeve, T., Shevchenko, A., Cudmore, S., Ashford, T., Mann, M., Griffiths, G., and Krijnse Locker, J. (1996). Identification of the major membrane and core proteins of vaccinia virus by two-dimensional electrophoresis. J. Virol. 70, 7485-7497.

9. Kolli, S., Meng, X., Wu, X., Shengjuler, D., Cameron, C.E., Xiang, Y., and Deng, J. (2015). StructureFunction Analysis of Vaccinia Virus H7 Protein Reveals a Novel Phosphoinositide Binding Fold Essential for Poxvirus Replication. J. Virol. 89, 2209-2219.

10. Kremer, J.R., Mastronarde, D.N., and McIntosh, J.R. (1996). Computer Visualization of ThreeDimensional Image Data Using IMOD. J. Struct. Biol. 116, 71-76.

11. Krijnse Locker, J., Chlanda, P., Sachsenheimer, T., and Brügger, B. (2013). Poxvirus membrane biogenesis: rupture not disruption. Cell. Microbiol. 15, 190-199.

12. Mastronarde DN (2005) Automated electron microscope tomography using robust prediction of specimen movements. J Struct Biol 152: 36-51

13. Moss, B. (2015). Poxvirus membrane biogenesis. Virology 479-480, 619-626.

14. Martinez-Sanchez, A., Garcia, I., Asano, S., Lucic, V. and Fernandez, J.-J. (2014). Robust membrane detection based on tensor voting for electron toography. J. Struct. Biol. 186, $49-61$.

15. Pedersen, K., Snijder, E.J., Schleich, S., Roos, N., Griffiths, G., and Locker, J.K. (2000). Characterization of Vaccinia Virus Intracellular Cores: Implications for Viral Uncoating and Core Structure. J. Virol. 74, $3525-3536$.

16. Pettersen, E.F., Goddard, T.D., Huang, C.C., Meng, E.C., Couch, G.S., Croll, T.I., Morris, J.H. and Ferrin, T.E. (2021). UCSF ChimeraX: Structure visualization for researchers, educators, and developers. Protein Sci. 30:70-82.

17. Salmons, T., Kuhn, A., Wylie, F., Schleich, S., Rodriguez, J.R., Rodriguez, D., Esteban, M., Griffiths, G., and Locker, J.K. (1997). Vaccinia virus membrane proteins p8 and p16 are cotranslationally inserted into the rough endoplasmic reticulum and retained in the intermediate compartment. J. Virol. 71 , 7404-7420.

18. Satheshkumar, P.S., Weisberg, A., and Moss, B. (2009). Vaccinia Virus H7 Protein Contributes to the Formation of Crescent Membrane Precursors of Immature Virions. J. Virol. 83, 8439-8450.

19. Schindelin, J., Arganda-Carreras, I., Frise, E., Kaynig, V., Longair, M., Pietzsch, T., et al. (2012). Fiji: an open-source platform for biological-image analysis. Nat. Methods 9, 676-682. 
20. Sodeik, B., Griffiths, G., Ericsson, M., Moss, B., and Doms, R.W. (1994). Assembly of vaccinia virus: effects of rifampin on the intracellular distribution of viral protein p65. J. Virol. 68, 1103-1114.

21. Suarez, C., Hoppe, S., Pénard, E., Walther, P., and Krijnse-Locker, J. (2017). Vaccinia virus A11 is required for membrane rupture and viral membrane assembly. Cell. Microbiol. 19, e12756.

22. Szajner, P., Weisberg, A.S., Lebowitz, J., Heuser, J., and Moss, B. (2005). External scaffold of spherical immature poxvirus particles is made of protein trimers, forming a honeycomb lattice. J. Cell Biol. 170, 971-981.

23. Wallengren, K., Risco, C., Krijnse-Locker, J., Esteban, M., and Rodriguez, D. (2001). The A17L Gene Product of Vaccinia Virus Is Exposed on the Surface of IMV. Virology 290, 143-152.

24. Welsch, S., Müller, B., and Kräusslich, H.-G. (2007). More than one door - Budding of enveloped viruses through cellular membranes. FEBS Lett. 581, 2089-2097.

25. Xiao, C., and Rossmann, M.G. (2011). Structures of giant icosahedral eukaryotic dsDNA viruses. Curr Opin Virol 1, 101-109.

26. Zhang, Y., and Moss, B. (1992). Immature viral envelope formation is interrupted at the same stage by lac operator-mediated repression of the vaccinia virus $\mathrm{D} 13 \mathrm{~L}$ gene and by the drug rifampicin. Virology $187,643-653$.

\section{Tables}

Table l: Ability of various mutant $\mathrm{H} 7$ proteins to rescue the H7-phenotype

\begin{tabular}{lllll} 
H7-expression construct & IV & MV & Virosome & Network \\
\hline VACV H7ind+IPTG & + & + & - & - \\
\hline VACV H7ind-IPTG & - & - & + & + \\
\hline pEL-HA-H7 & + & + & - & - \\
\hline pEL-HA-H7-R109E & + & + & - & - \\
\hline pEL-HA-H7-R117E/K128E & + & + - & + +- & - \\
\hline pEL-HA-H7-K108E/R109E/K112E & - & - & + & + \\
\hline pEL-HA-H7-119-146 & - & - & + & + \\
\hline pEL-HA-H7-1-114 & - & - & + & +
\end{tabular}

\section{Figures}



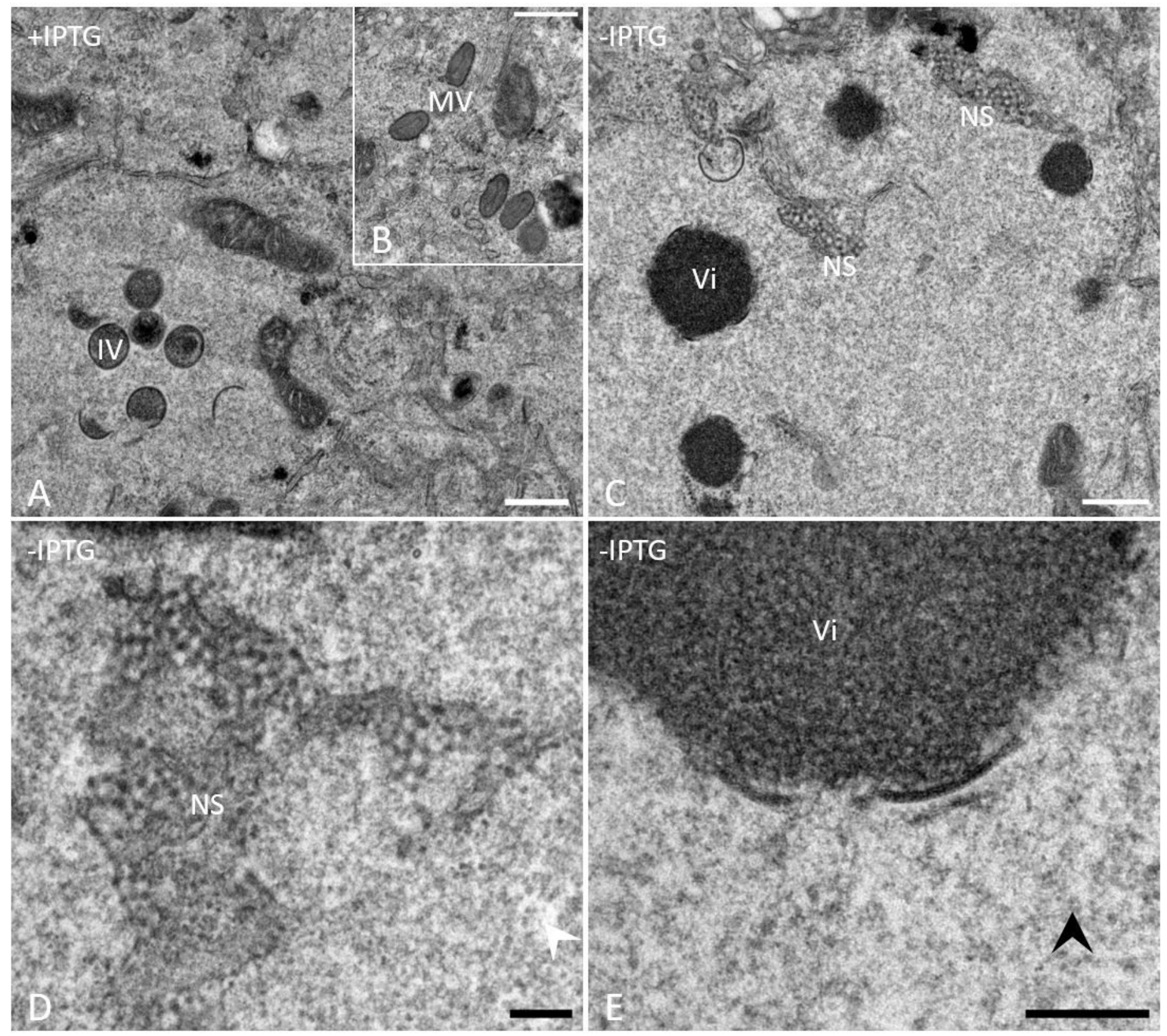

\section{Figure 1}

In the absence of $\mathrm{H} 7$ two prominent structures accumulate in infected HeLa cells. HeLa cells were infected and fixed 12 hours post infection, embedded in epoxy resin, sectioned and imaged by TEM. Infected cells with IPTG $(A, B)$ show wild-type phenotype with immature virus (IV) and mature virus (MV). Without IPTG infected cells display two prominent structures (C): network-like structures (NS) and electron dense areas collecting core protein, virosomes ( $\mathrm{Vi})$. At higher magnification the NS is composed of electron-dense dots arranged in a regular pattern ( $D$; white arrowhead). The electron dense area (virosome, Vi) in E displays short crescents-like structures (black arrowhead). Scale bar: 500nm (white), 200nm (black). 


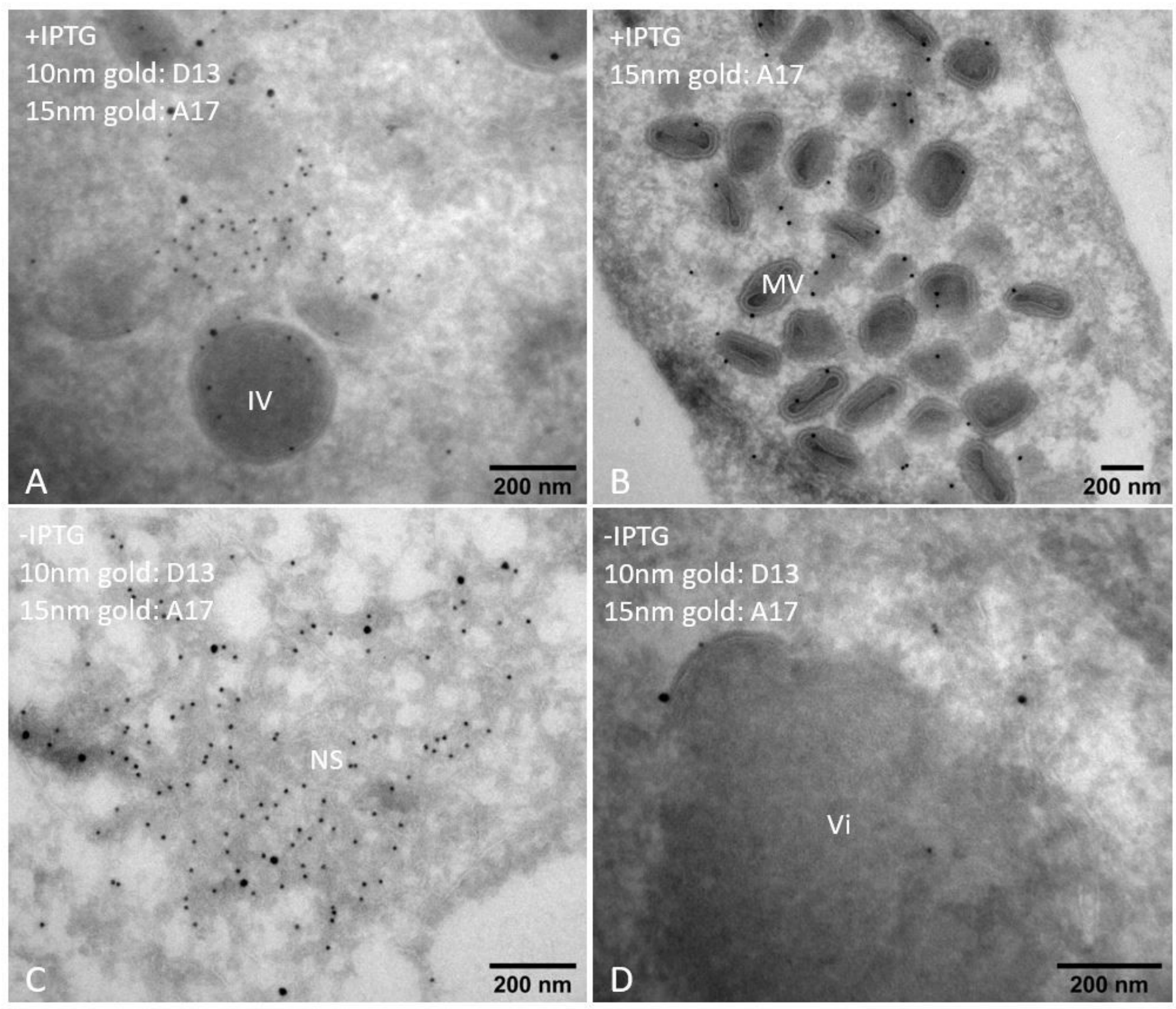

Figure 2

EM-immunolabeling shows the accumulation of D13 on the network structure. HeLa cells were infected as for figure 1 and embedded for EM-immuno-labeling. With IPTG immature virus (IV) and mature virus (MV) show labeling for A17 and D13 (A and B). (C) Double-labeling of the NS that accumulates without IPTG, showing abundant labeling for D13 and labeling for A17 on membranes, indicating the presence of the ER. The short crescents that accumulate on the electron-dense structures $(\mathrm{Vi})$ in D are labeled by D13 and A17 (D). Scale bar: 200nm. 

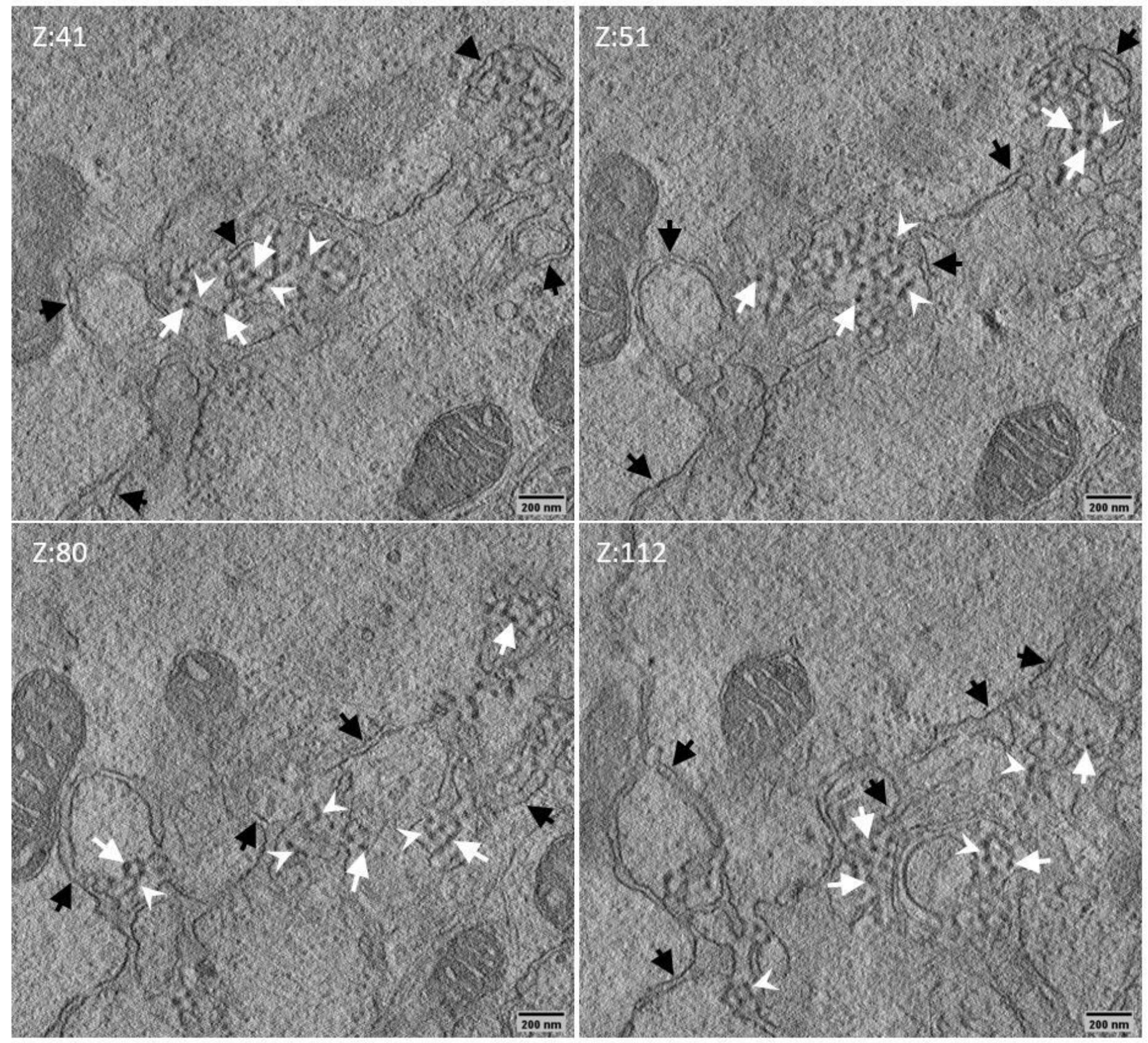

\section{Figure 3}

The D13 network structure is intimately associated with the ER. HeLa cells infected without IPTG were prepared as in figure 1. Sections, 750nm in thickness, were subjected to STEM tomography. The figure displays four slices of the reconstructed tomogram. It shows the electron-dense spots (white arrows) displaying a regular pattern. Slice by slice inspection also shows that the spots are connected by thin structures, likely thin membrane tubes (white arrow head; movie 1). The network structure is associated with, and surrounded by, the ER (black arrows). The slices are taken from the supplemental movie 1 . Scale bar: $200 \mathrm{~nm}$. 


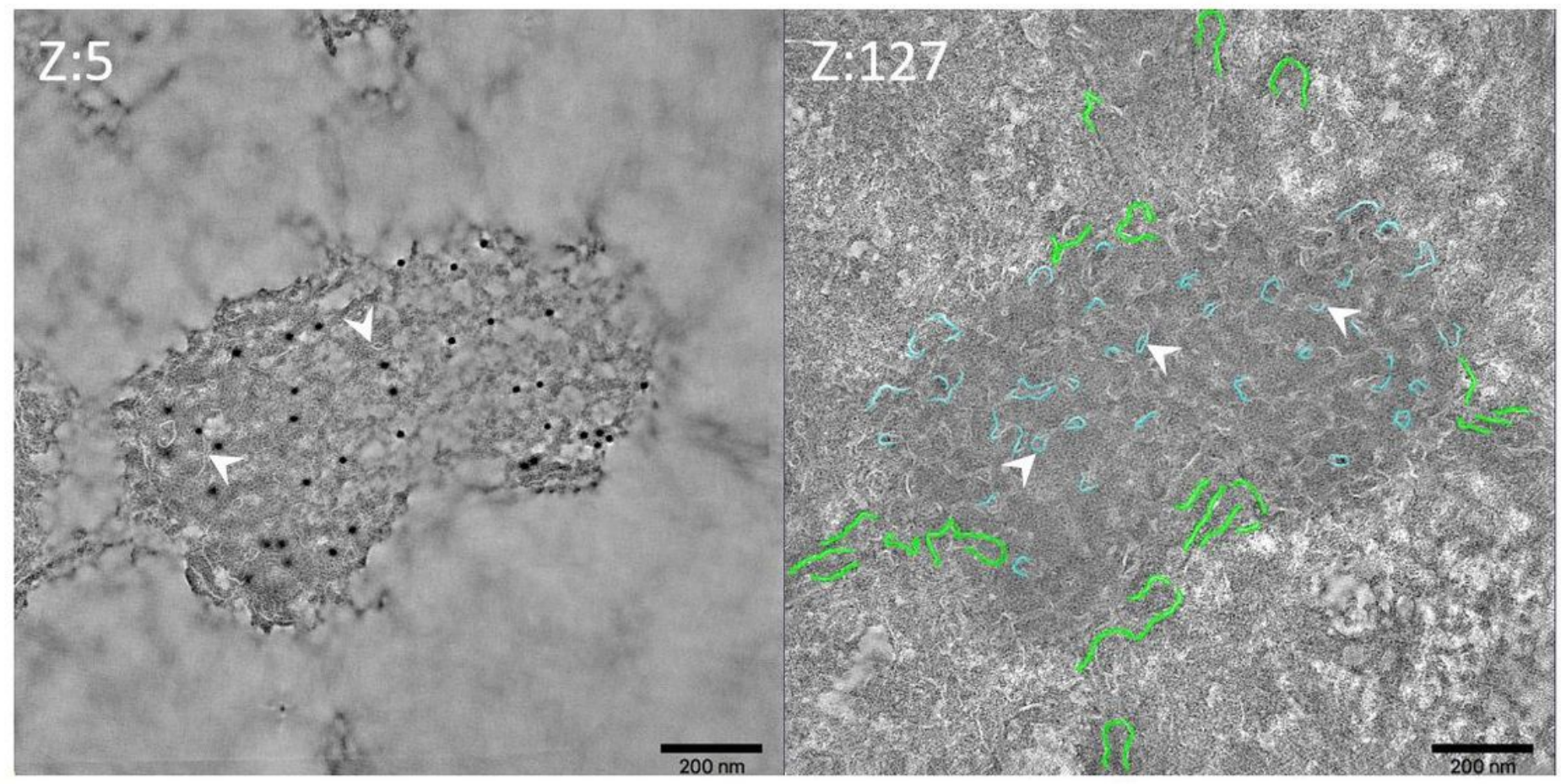

\section{Figure 4}

Short membrane tubes prominently accumulate on the D13 network. HeLa cells infected without IPTG were prepared as for figure 2. Sections, $150 \mathrm{~nm}$ in thickness, were labeled with anti-D13 and subjected to dual-axis tilt series acquisition by transmission EM. Two slices of the reconstructed tomogram; the surface of the section (Z:5) displays anti-D13 labeling and identifies the D13-positive network structure in Tokuyasu sections. The slice Z:127 illustrates that the D13 network is filled with short membrane tubes (white arrowheads) that appear as white lines due to the negative contrasting in the Tokuyasu method. A subset of the short membrane tubes is rendered in light blue, while the ER cisternae that accumulate close by are rendered in green. The slices are taken from the supplemental movie 4. 

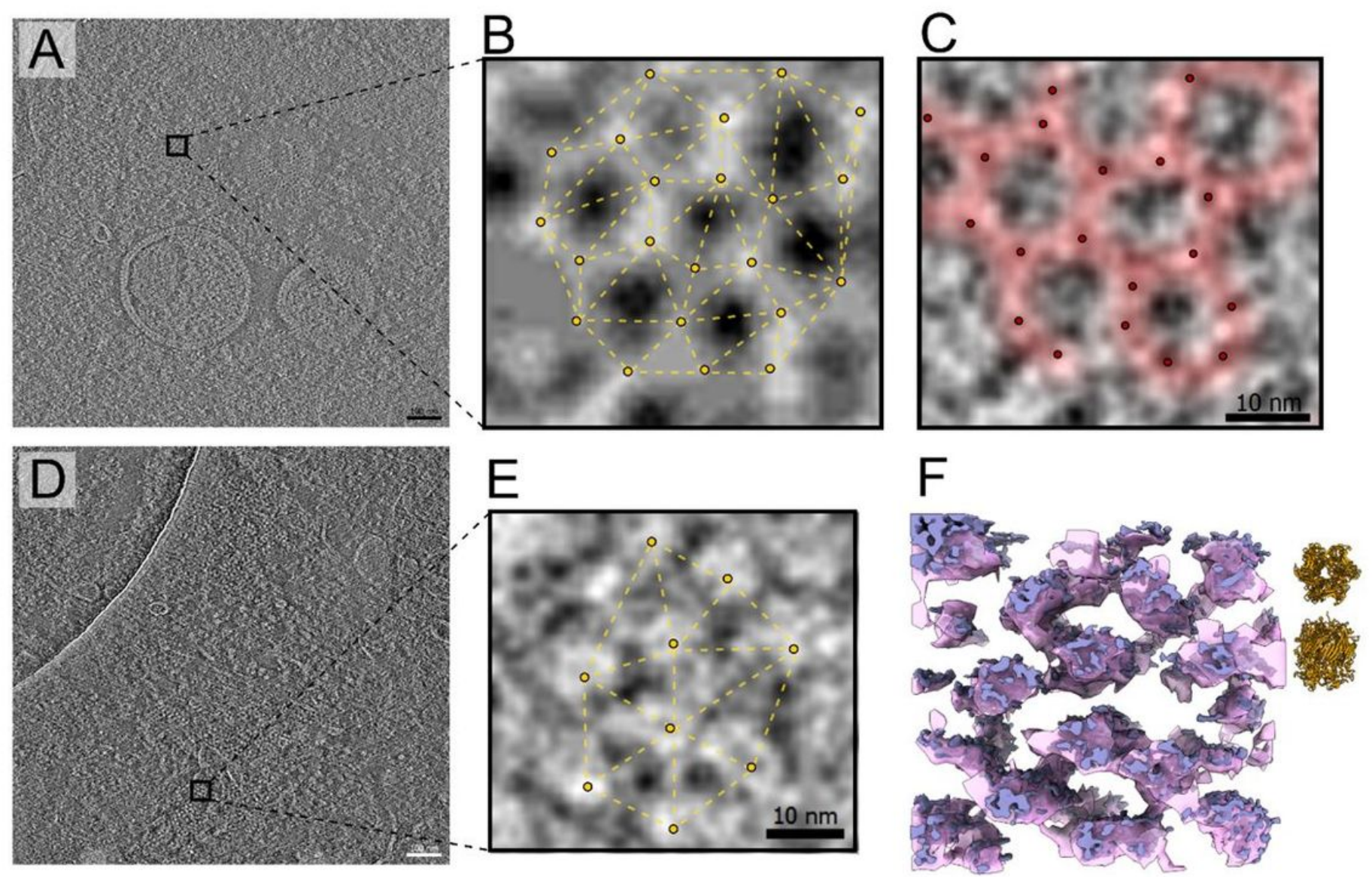

\section{Figure 5}

\section{Honeycomb lattice formation is impaired in the absence of $\mathrm{H7}$ synthesis.}

HeLa cells were infected and prepared as for figure 2 and $70 \mathrm{~nm}$ thick sections immuno-labeled with antiD13 followed by protein A coupled to $10 \mathrm{~nm}$ gold, prior to plunge freezing. A shows a slice of a tomogram of HeLa cells infected in the presence of IPTG (movie 5) with two IVs and a zoom (B and C) of the boxed area in $A$. The contrast is inverted for all images. In B and C the honey-comb pattern of D13 is apparent. In B the information is shown after application of the scale-space filter (Martinez-Sanchez et al., 2014) to fully reveal the hexagonal arrangement of the D13 trimers (yellow dots in B, black dots in C, Supplemental Figure 3). D) a slice of a tomogram of HeLa cells infected without IPTG. A zoom of the boxed area in D is shown in E and F. Individual D13 trimers (yellow dots in E) located at a greater distance from each other (Delaunay triangulation distance $=13.75 \pm 2.67 \mathrm{~nm}$ ), failing to arrange in the typical honeycomb lattice (Delaunay triangulation distance $=10.80 \pm 3.23 \mathrm{~nm}$, Supplemental Figure 3). In F the D13 trimers are rendered in violet and pink based on their original intensities and their gaussian filter respectively using different isosurface thresholds. In the extreme upper right corner of $F$, two different orientations (top \& side view) of the D13 atomic structure (pdb id: 2ygb) in yellow cartoon representation, as size reference. 


\section{Supplementary Files}

This is a list of supplementary files associated with this preprint. Click to download.

- Supplementaldata.docx

- Tonnemacheretal.supplementalfigures.docx

- movie1.avi

- movie2.mov

- movie3.mov

- movie4.mov

- movie5.avi

- movie6.avi 